\title{
Integrationspotential siliziumbasierter Sensoren mittels technologischer Plattformen und Module
}

\author{
A. Steinke, D. Preuß, M. Hintz, O. Brodersen, S. Völlmeke \\ CiS Forschungsinstitut für Mikrosensorik und Photovoltaik GmbH, 99099 Erfurt, Germany \\ asteinke@cismst.de
}

\begin{abstract}
Einleitung
Moderne Produkte der Mikrosystemtechnik sind heute gekennzeichnet durch einen hohen Integrationsgrad, Packungsdichte aber auch hoher Systemtiefe. Auf sehr hohe Stückzahlen mit extremen Kosten- und Qualitätsdruck ausgerichtete Produkte im Automotiv-, Weiße-Ware- oder ITSektor wie bspw. Druck- und Beschleunigungssensoren erfordern spezifisch darauf entwickelte Herstellungs-Technologien. Demgegenüber besteht das Interesse von KMU ebenfalls von den Stateof-the-Art Technologien zu partizipieren, aber unter dem Aspekt mittlerer Stückzahlen und spezifischer Anforderungen (bspw. Temperatur, Selektivität, ...). Eine Lösung besteht in technologischen Plattformen, die anstelle einer Vollintegration der Sensorsignal- Komponenten die Hybridintegration der Komponenten wie Filter, Erkennungssystem, Transducer (und Signalvorverarbeitung) basieren und damit einen wesentlich größeren technologischen Spielraum besitzen.
\end{abstract}

\begin{abstract}
Vollintegration vs. Hybridintegration
"More Moore" und "More than Moore" Strategien spielen zunehmend eine Schlüsselrolle innerhalb der MikroNanoSystemtechnologien. Die technologischen Prozesse/Teilschritte (More Moore) sind bezüglich ihrer Bedeutung sowohl systemrelevant für modulare Komponenten als auch für die Systemintegration. Die technologischen Trends, beschrieben bspw. im "White Paper" [1] (Abbildung 1) folgen dem Trend der Hochintegration, erlauben aber auch die Entwicklung von Modulen, wie sie für die Wertsteigerung von Nutzen sind.
\end{abstract}

In Verbindung mit Key Enable Technologien (KET) [2] sind neue Märkte primär im Fokus von Forschung und Entwicklung. [3]

Die "More than Moore"-Strategie in Verbindung mit einer CMOS- und MEMS-Technologie zielt insbesondere auf „system-on-chip“-Lösungen. Dabei müssen aber die Einschränkungen bedingt durch den CMOS-Prozess bspw. Temperatur/Umgebungsbedingungen, bei den speziellen Applikationen, Kosten vs. Stückzahlen berücksichtigt werden.

Auf der anderen Seite haben aber insbesondere kleine und mittlere Unternehmen ein zunehmendes Interesse an Sensoren, die in immer raueren Umgebungsbedingungen arbeiten müssen (in-line), bei denen auftretende Artefakte durch eine intelligente Fehlerkompensation beherrscht werden, die state-of-the-art-Technologien nutzen, um den Forderungen nach Selektivität und Sensitivität zu entsprechen, die aber auch bezahlbar in

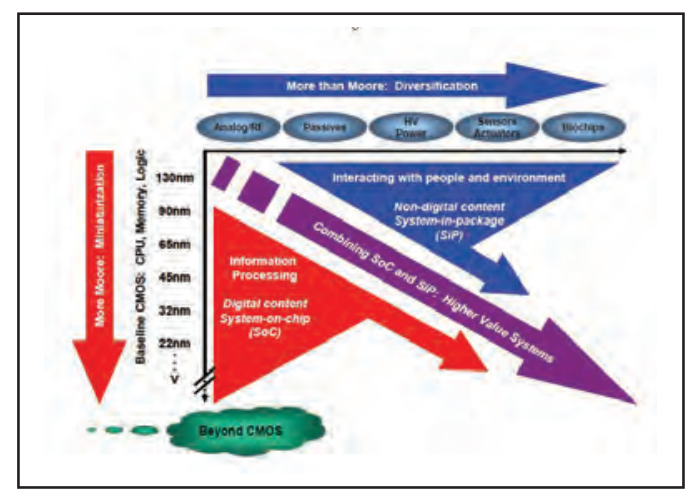

Abbildung 1: "More Moore"- vs. "More than Moore"Strategie [1]

kleinen und mittleren Stückzahlen bereitgestellt werden können.

Diese Forderungen sind aber in ihrer Komplexität mit einer CMOS-Integration aller Sensorkomponenten schwer zu realisieren.

Abbildung 2 illustriert die Komplexität des Sensorsignalsystems unter dem Gesichtspunkt spezifischer technischer und technologischer Parameter, wenn eine Vollintegration das Produktziel ist.

Unabhängig ob als Frontend- oder als Backend-Prozess, müssen technologische Einflüsse (bspw. Prozesstemperatur) in weitaus größerem Maße für die einzelnen Sensorsignal-Komponenten berücksichtigt werden, da sie sowohl deren technologische Spielräume einschränken, als auch die Signalmodulation des Erkennungssystems (bspw. sensitive Schicht) und des/der Transducer(s) (bspw. Photoempfänger) 
einschränken (1). Darüber hinaus können sich elektrische und thermische Rückkopplungen während des (elektrischen) Betriebes des Sensorsystems, ausgelöst durch den
Leistungsverbrauch der Signalverarbeitung und elektronischen Schnittstellen, negativ auf die Genauigkeit und Langzeitstabilität des Erkennungssystems auswirken (2).

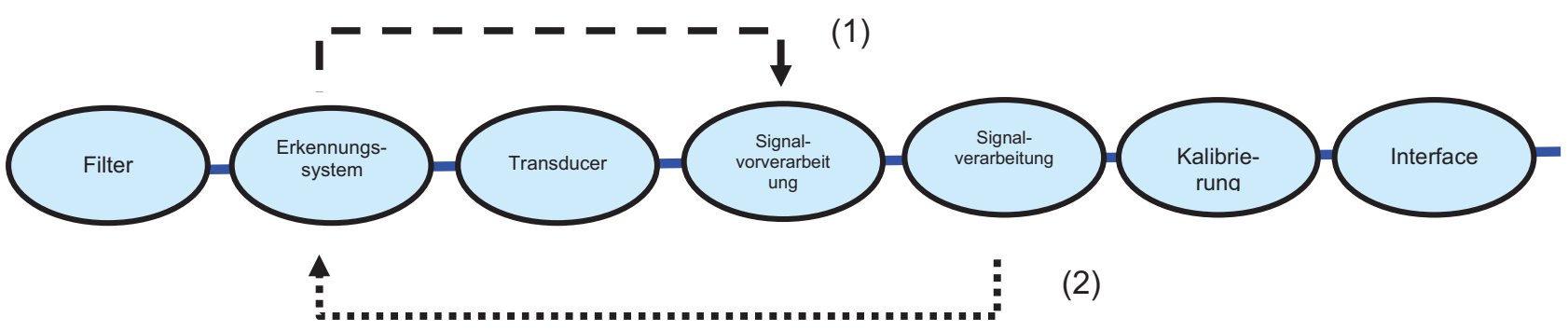

Abbildung 2: Sensorsignalkomponenten

Als alternative Strategie, um den Forderungen der KMUs nachzukommen, präsentieren die Autoren technologische Plattformen, die sich auf die Sensorsignal-Komponenten Filter, Erkennungssystem, Transducer, Signalvorverarbeitung konzentrieren (Abbildung 3).

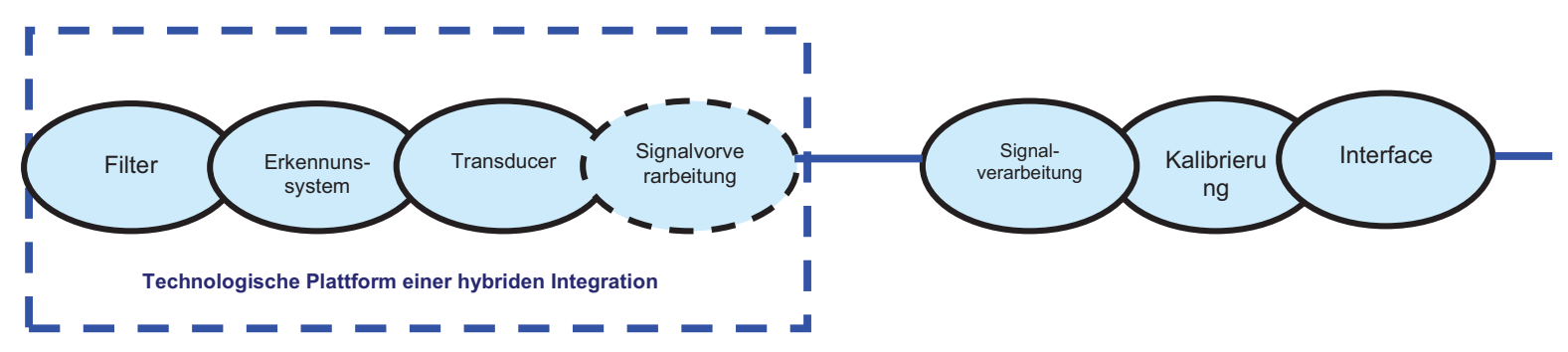

Abbildung 3: Sensorkomponenten der technologischen Plattform

Eine Plattformstrategie erhöht deutlich den technologischen Handlungsspielraum, schränkt aber auch nicht wesentlich die technologischen Optionen eines Batch- und Mikro-Assembly-Prozesses [4] ein.
In Verbindung mit batchprozesskompatiblen technologischen Modulen wird eine hohe Flexibilität sowohl technologisch als auch produktseitig erreicht (Abbildung 4).

\section{Technologische Module - Technologische Plattformen}

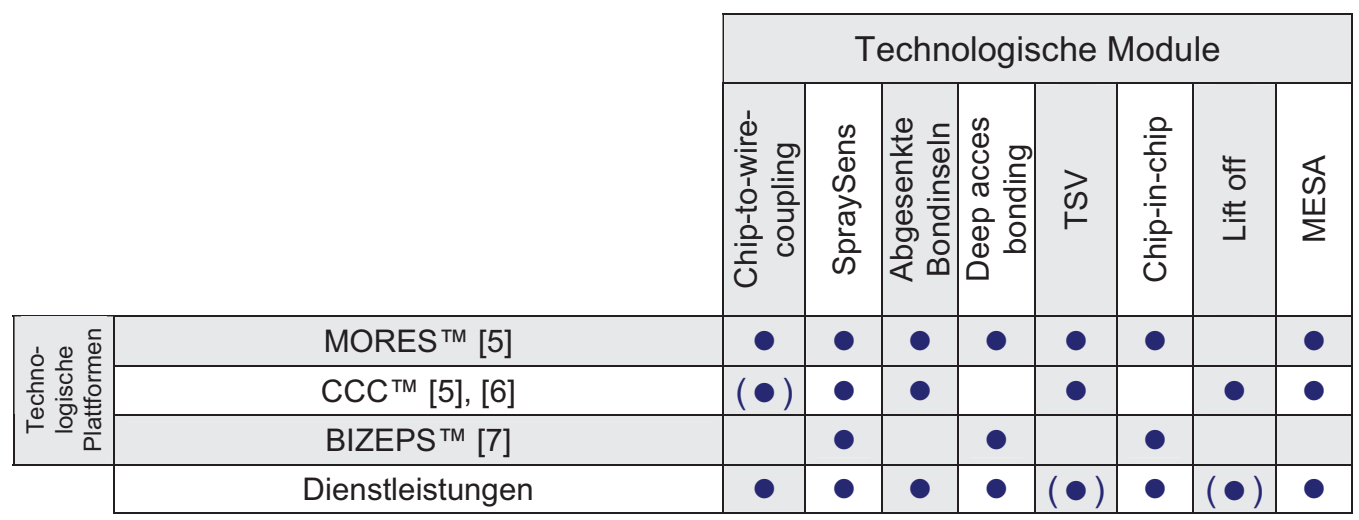

Abbildung 4: Übersicht technologischer Plattformen und technologischer Module (Auswahl)

(• vorhanden; (•) in Entwicklung) 
Das Herzstück der technologischen Plattformen sind die silizium-integrierten Transducer (optoelektronisch, impedimetrisch, piezoresistiv, Schalter). Diese einzelnen oder auch hochintegrierten Transducer (Multisensoren als Array gleicher oder unterschiedlicher 2D/3D-Transducer) werden mittels technologischer (kompatibler) Module bzgl. Sensorsignal-Komponenten bzw. Interface-Komponenten in ihrer Wertschöpfung ergänzt. Dabei werden als SensorsignalKomponenten Filter, Erkennungssysteme und Signalvorverarbeitung betrachtet, während für Interface-Komponenten die technologischen Schnittstellen analysiert werden (bspw.

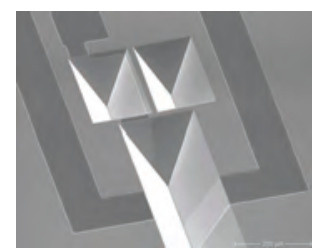

Chip-to-wire- Montage

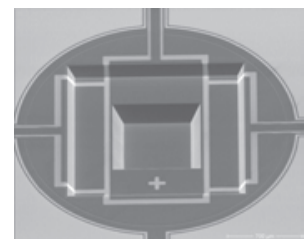

Abgesenkte Bondinseln

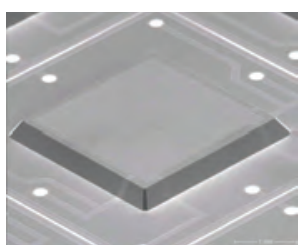

MESA

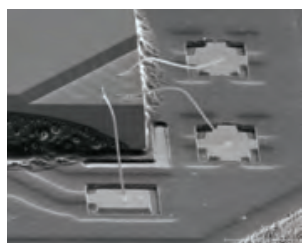

Deep access bonding

Durchkontaktierungen, SprayCoating, Chip-inChip). Dabei basieren derartige Plattformen auf Evaluierungen gem. ISO 9001 und sind u.a. auch durch Design-Regeln beschrieben.

Abbildung 5 zeigt eine Auswahl von technologischen Modulen. Diese erweitern das Applikationspotential der technologischen Plattformen (Abbildung 4).

Basierend auf dieser Strategie wurde die MORES ${ }^{\mathrm{TM}}$ Plattform (microoptical remission sensor) entwickelt, welche für verschiedene optische Prinzipien wie Reflektion, Colorimetrie, Fluoreszenz, Interferenz geeignet ist.

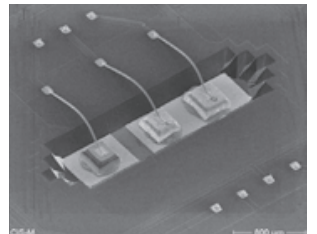

Chip-in-Chip

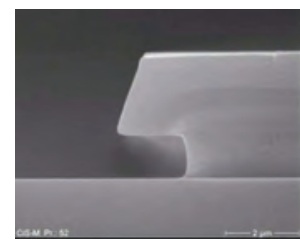

lift off

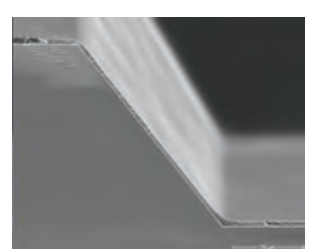

SpraySens

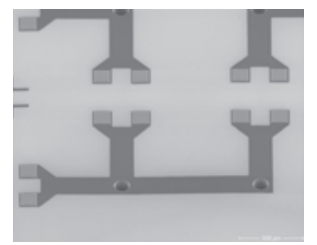

TSV

Abbildung 5: Auswahl technologischer Module

Die Kombination von Transducern und technologischen Modulen wurden getestet und gemäß ISO 9001 und spezifischen Anforderungen der Kunden qualifiziert.

\section{D Strukturierung}

Die Herstellung mikromechanischer Sensoren als auch Interposer erfordert oftmals eine dreidimensionale Tiefenstrukturierung des Siliziumwafers.

Dazu kommen in vielen Anwendungen anisotrope nasschemische Ätzungen mit Böschungswinkeln von $54,7^{\circ}, 45^{\circ}$ und $90^{\circ}$ sowie isotrope nasschemische und elektrochemische Ätzungen zum Einsatz. Andere Applikationen erfordern senkrechte bzw. sehr steile Flanken mit nahezu beliebigen lateralen Konturen, die mit Hilfe des ICP DRIE Plasmaätz-Verfahrens hergestellt werden. Hiermit kann auch, gegenüber den nasschemischen Verfahren, die Packungsdichte von Strukturen, wie z.B. Durchkontaktierungen, deutlich erhöht werden. Im weiteren Herstellungsprozess ist es häufig erforderlich, dielektrische (z.B. Si3N4) und leitfähige (z.B. AISi) Schichten auf den erzeugten Topographien abzuscheiden und zu strukturieren. Hier versagt die konventionelle Photolithographie, da sich mittels Spin-Coating keine konformen und kontinuierlichen Lackschichten über die Ätzflanken erzielen lassen.

Auf 3D-Oberflächen mit stufenweise ebenen, schrägen, bis hin zu extrem steilen Topologien hat sich das Spray-Coating von Photoresist als vielfältig einsetzbare und kostengünstige Technik etabliert. Hier gelang es in den letzten Jahren, die Konformität der Photoresistbedeckung über die Topographien hinweg deutlich zu steigern (Abbildung 5). In Verbindung mit einer neuartigen MikrooptikMask-Aligner-Belichtung lassen sich gleichzeitig Oberflächenstrukturen bis zu $2 \mu \mathrm{m}$ und Tiefenstrukturen bis zu ca. $10 \mu \mathrm{m}$ in 250 $\mu \mathrm{m}$ Tiefe auflösen (Abbildung 6). Mit Anpassung der Metall- und Isolationssysteme lassen sich diese Technologiemodule auch um eine Under-Bump-Metallisierung erweitern. 


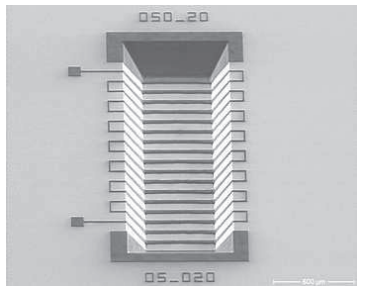

Abbildung 6: Auflösungsteststruktur

\section{Assembly}

Das Beispiel des optischen Nivellierungssensors zeigt die Vorteile der Kombination einer 3D-SiliziumWaferstrukturierung und modernster Montagetechnologien. Kavitäten im SiliziumBulk ermöglichen die Implantation applikationsspezifischer Strahlerelemente (LED, VCSEL) als Dies, die aber auch gleichzeitig durch ein (hoch)integriertes Array von Detektoren umgeben sind. Hierfür wird ein hochpräziser automatisierter Die-BondProzess eingesetzt, welcher die Klebung mittels elektrisch leitfähiger oder isolierender Materialien erlaubt,

Abgesenkte Bondinseln in Verbindung mit extrem flachen Drahtbrücken sind die Basis für innovative miniaturisierte Module, die sich letztendlich durch eine planare Oberfläche (Abbildungen 5 und 9) auszeichnen. Bondungen mit Gold- oder Aluminium-Drähten

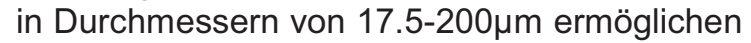
einen breiten Anwendungsbereich und bieten technologische Alternativen für unterschiedliche Betriebsbedingungen.

Die flexible Nutzung verschiedener Montagetechnologien (Fineplacerß, Mikromanipulatoren, SMD-Bestücker etc.) ermöglicht weiterhin die hochpräzise Montage von optischen (Linsen, Blenden), mechanischen und elektrischen Komponenten. Neben der Kontaktierung durch Drahtbondprozesse werden auch verschiedene Flip-Chip-Technologien eingesetzt. Studbumpsoldering (auch flussmittelfrei) als auch Thermosonic-Prozesse ermöglichen als (hybride) Alternative zur Siliziumvollintegration die Realisierung von extrem kompakten Modulen mit minimaler Baugröße und minimalen Signalstrecken.

Für die verschiedenen Anwendungen werden gezielt Verguss-, Unterfüll- und Klebematerialien ausgewählt, um zuverlässige, störunempfindliche Aufbauten mit minimalen Querempfindlichkeiten und hoher Signalgüte sicherzustellen, Eigenschaften wie bespielsweise biomedizinische Kompatibilität, thermischer Ausdehnungskoeffizient, Feuchtebeständigkeit, optisches Verhalten aber auch die automatisierte Verarbeitbarkeit sind hierbei wichtige Kriterien.

Abbildung 7 zeigt eine Integrationsform von MESA-Silizium-Chips (Beispiel: $\mathrm{CCC}^{\mathrm{TM}}$ Plattform) und einer Flip-Chip-Montage. Dies stellt eine Möglichkeit der physischen Entkopplung der Kontaktsysteme von der Umwelt dar und ist als ein Schritt auf dem Weg bis hin zu Durchkontaktierungen zu sehen. Die Kombination von TSV (Trough Silicon Vias) und/oder 3D strukturierten Substraten kann auch die Basis für den Einsatz als Interposer für hochintegrierte Hybridsysteme sein und eröffnet damit einen weiteren weiten Bereich möglicher Funktionalitäten.

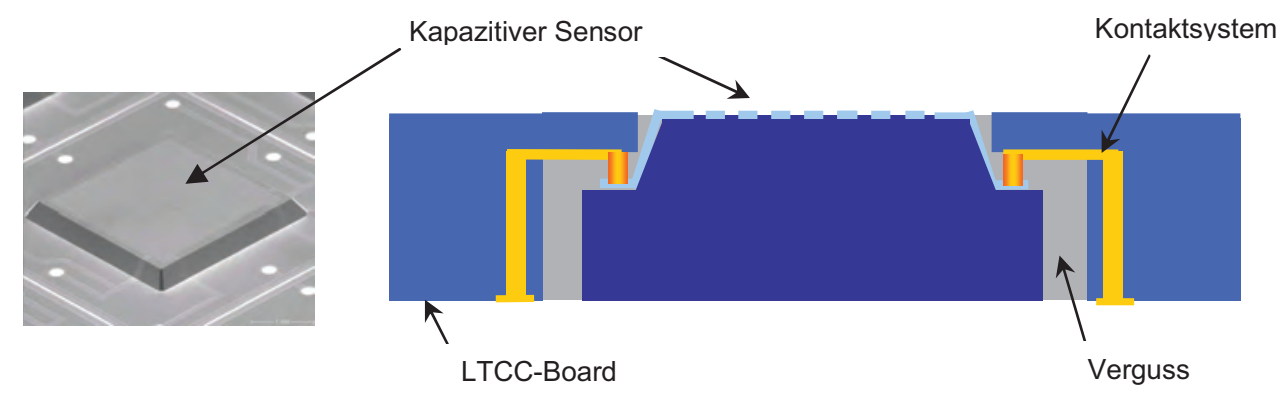

Abbildung 7: Chip mit erhabenem Erkennungssystem zur Flip-Chip-Montage in ein LTCC-Substrat 


\section{Applikation}

In Forschung und Entwicklung ist eine präzis ausgerichtete Referenzposition oft von entscheidender Bedeutung. Beispielsweise ist das Wägen von Medikamenten eine Herausforderung, dieses immer sicherer und zuverlässiger vornehmen zu können, um damit Dosierungen exakt zu optimieren und zu reproduzieren. Dies gilt sowohl für die Einnahme von Medikamenten als auch für den Herstellungsprozess an sich [8]. Minimale Abweichungen vom Zielwert können bei der Herstellung von Medikamenten fatale Konsequenzen haben.

Die CiS Forschungsinstitut für Mikrosensorik und Photovoltaik $\mathrm{GmbH}$ hat in Kooperation mit der Sartorius Weighing Technology $\mathrm{GmbH}$ und Stahl Präzisionsionslibellen KG den elektronischen Nivellierungssensor eLiSe entwickelt.

Der Sensor eLiSe ermöglicht eine automatische Erkennung von Neigungsänderungen und wandelt diese in ein analoges Signal. Dieses steht für Korrekturmaßnahmen (mechanische als auch automatische Korrektur) zur Verfügung.

Grundlage für diesen Nivellierungssensor bildete die technologische Plattform MORES ${ }^{\mathrm{TM}}$.
Entsprechend der Aufgabenstellung, die Position der Gasblase in einer Dosenlibelle zu bestimmen, wurde das Design der MORESBaugruppe optimiert und bereits auf Kompatibilität getestete technologische Module in den Prozessablauf integriert (Abbildung 5). Die Strategie der Nutzung von Ergebnissen vorangegangener FuE-Arbeiten im Rahmen der Entwicklung ermöglichte es, dass in relativ kurzer Zeit erste Muster entwickelt werden konnten. Dies beschleunigte den Zeitraum bis zur Markteinführung signifikant, zumal auch diese Strategie für den Kunden ein Potential weiterer Entwicklungen (Skalierung, innovative Lösungen) aufzeigte.

Die Eigenschaften der Dosenlibelle (Abbildung 8) bestimmen den Messbereich und die Empfindlichkeit des Gesamtsystems. Das opto-elektronische Abtastmodul mit seinem MORES ${ }^{\mathrm{TM}}$-Modul als Herzstück richtet das emittierte Licht in die Dosenlibelle. Das von der Gasblase diffus reflektierte Licht wird von dem 4-Qudranten-Empfänger erfasst. Aus den Einzelsignalen kann die exakte Position der Blase ermittelt werden.

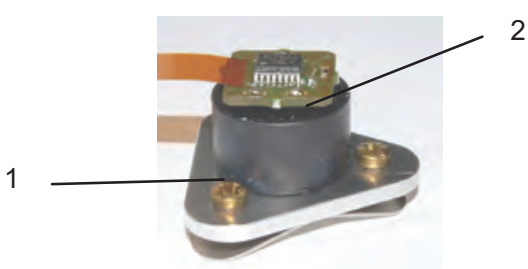

Abbildung 8: Nivellierungssensor

Abbildung 9 zeigt den Prozessablauf der MORES $^{\mathrm{TM}}$ Plattform am Beispiel des Nivellierungssensors. Dabei sind neben den Standardprozessen der MORES ${ }^{\mathrm{TM}}$ Plattform, die ergänzenden technologischen Module erkennbar, wie sie für die Entwicklung der Sensorbaugruppe erforderlich sind.

\section{Zusammenfassung}

Die Strategie des CiS Forschungsinstitutes, insbesondere für die Entwicklung von Produktinnovationen für kleine und mittlere Unternehmen, basiert sowohl auf der "More Moore"- als auch auf der "More than Moore"Strategie. Dabei bezieht sich die Nutzung von Forschungs- und Entwicklungsergebnissen der „More Moore“-Strategie nicht auf die der
Hochintegration bzw. Wafergröße, sondern auf die Performance der technologischen Prozesse. Verfahren wie Inductive Coupled Plasma Strukturierung (ICP), Silicon Fusion Bonden (SFB) als auch Atomic Layer Deposition (ALD) sind bereits heute aus der siliziumbasierten Mikro-Nano-Sensorik nicht mehr wegzudenken. Im Gegensatz zu den CMOS-Prozessen bieten die in diesem Beitrag beschriebenen technologischen Plattformen, wie z.B. MORES ${ }^{\mathrm{TM}}, \mathrm{CCC}^{\mathrm{TM}}$ und BIZEPS ${ }^{\mathrm{TM}}$, einen größeren technologischen Spielraum hinsichtlich "high performance"-Sensoren.

Das Herzstück dieser Plattformen sind die auf die Anforderungen des Kunden ausgerichteten Technologien der Transducer.

Zur Generierung von Signalen zur Fehlerkompensation oder zur Eigendiagnose 
ermöglichen sie aber auch die Integration von Multi-Sensoren (hier: optoelektronisch, impedimetrisch, piezoresistiv) sowohl in 2- als auch 3 dimensionaler Anordnung. Mittels technologiekompatibler Module ist sowohl eine physische Entkopplung des technologischen Prozesses als auch des Signalsystems von der
Umwelt für die verbesserte Langzeitzuverlässigkeit möglich. .

Die Effizienz derartiger Plattformen in Verbindung mit dem Potential technologischer Module liegt insbesondere auch darin, dass für diese Module bereits Testergebnisse zur Zuverlässigkeit vorliegen und damit nachhaltig das „Proof of Concept“ bestätigen.

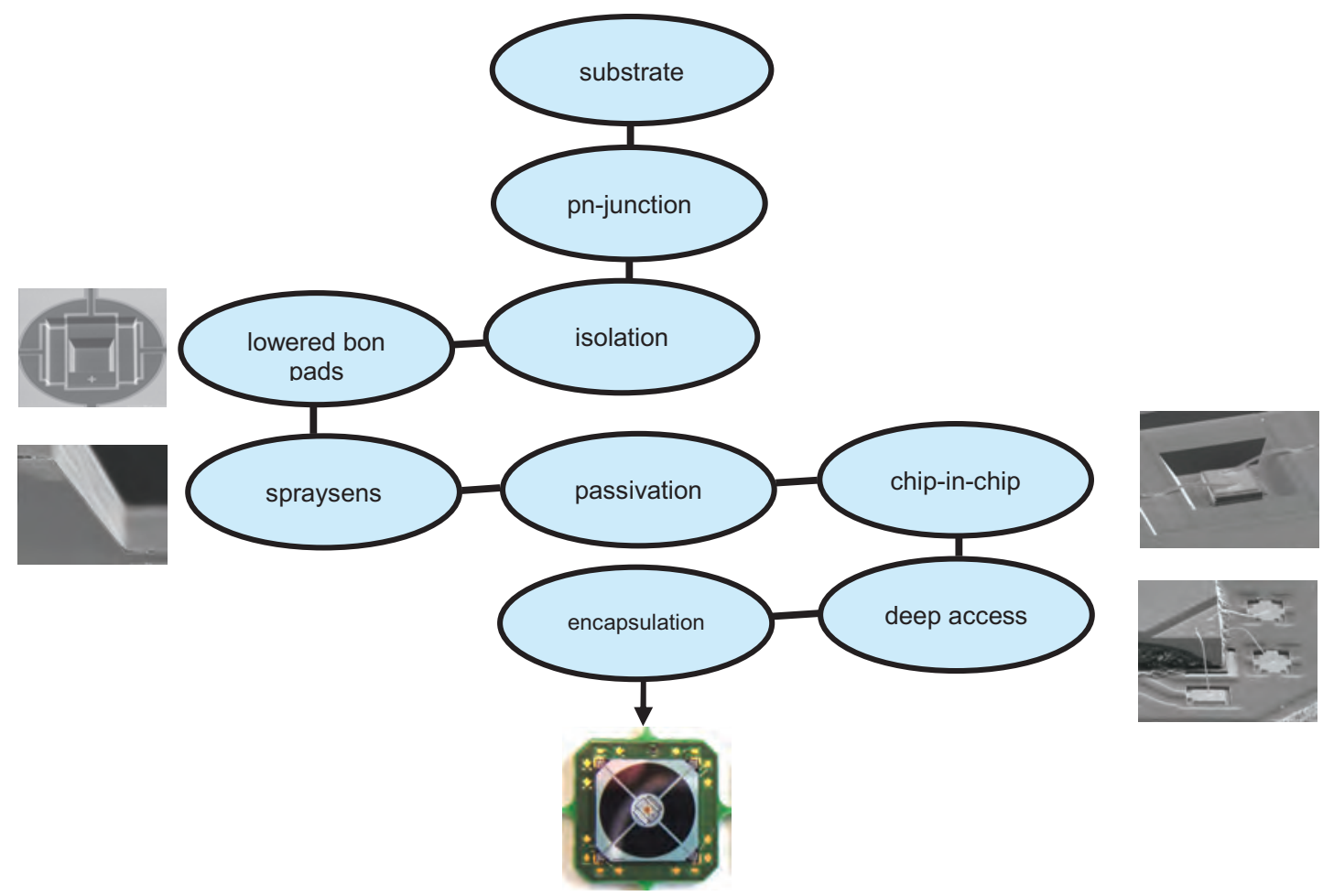

Abbildung 9: Prozessablauf der MORES ${ }^{\mathrm{TM}}$ Plattform am Beispiel des Nivellierungssensors

\section{Quellen}

[1] More-than-Moore-Roadmapping Update, ITRS public conference, San Francisco, July 13, 2010

[2] High-Level Expert Group on Key Enable Technologies, Final Report, June 2011

[3] Schwarz, U., Schmidt, K.; Integration of CMOS and MEMS processes as part of X-FAB's "More than Moore" Modularity; Smart System Integration, Zurich, 21-22 March 2012

[4] Robert, D.; Partnership Models for MEMS Business; SEMICON WEST 2012

[5] Brodersen, O., Semmler, K., Steinke, A.; Smart System Technologies for SME (and lower quantities/pieces), EPoSS Annual Forum 2010, Lisbon, 7-8 October 2010

[6] Steinke, A., March, B., Heinze, D., Hansch, H.; The CCC*-dew point sensor-as a concrete example of design and industrial realization of monolithic transducers;
SENSOR EXPO `98, Chicago, 3-10 October 1998

[7] Frank, Th., Steinke, A.; Electric power- free smart sensor platform based on smart sensitive materials, EPoSS Annual Forum 2011, Barcelona, 5-7 October 2011

[8] CiS Forschungsinstitut für Mikrosensorik und Photovoltaik $\mathrm{GmbH}$, Applikationszentrum Mikrooptische Systeme: Perfekt ausgerichtet. Elektronischer Libellensensor eLiSe. Erfurt, 2009. - Firmenschrift

MORES ${ }^{\mathrm{TM}}$ (microoptical remission sensor),

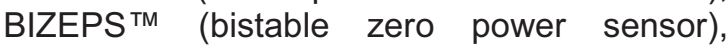
$\mathrm{CCC}^{\mathrm{TM}}$ (condensate controlled capacitance) are registered trademarks of the CiS Forschungsinstitut für Mikrosensorik und Photovoltaik $\mathrm{GmbH}$. 